\title{
Sterile Pericarditis Leading To Cardiac Temponade from Intrapericardial Foreign Body 3 Months Following Gunshot Injury to Chest: Syndrome of Acute Intermittent Pericarditis
}

\author{
Dr. R. M. Mathur ${ }^{1}$, Dr. Sanjeev Devgarha ${ }^{2}$, Dr. Dheeraj Sharma ${ }^{3}$, \\ Dr. Hema Chaudhary ${ }^{4}$ \\ ${ }^{I}$ (Professor and Head Department of cardiothoracic and vascular surgery, S.M.S Medical college, Jaipur, \\ Rajasthan, India) \\ ${ }^{2}$ (Associate Professor, Department of cardiothoracic and vascular surgery, S.M.S Medical college, Jaipur, \\ Rajasthan, India) \\ ${ }^{3}$ (Senior Resident, Department of cardiothoracic and vascular surgery, S.M.S Medical college, Jaipur, \\ Rajasthan, India) \\ ${ }^{4}$ (Senior Resident, Department of Radiodiagnosis, L.L.R.M Medical College, Meerut ,U.P. ,India)
}

\begin{abstract}
Patients with bullets in the pericardial sac without myocardial injuries are rare and most commonly are associated with significant trauma. The diagnosis of an intrapericardial foreign body can be difficult. Its removal is always indicated because it prevents pericarditis either sterile or infectious with potential for other significant complications. The syndrome of acute intermittent pericarditis from a foreign body within the pericardium is rarely seen except during time of war. Recently the authors treated a patient with a bullet in the pericardium three month following gunshot injury. During this period the patient developed sterile pericarditis and tamponade which necessitated emergency pericardiectomy and removal of the bullet. This experience and a brief review of the pertinent literature follows.
\end{abstract}

Keywords: Foreign body, Gunshot, Intrapericardial, Sterile Pericarditis, Pericardium injuries, Temponade.

\section{INTRODUCTION}

With no associated injury in the myocardium the finding of bullet alone in the pericardial cavity is rare. In this condition the evolution is unpredictable, because some of the patients remain asymptomatic, pericarditis occurs in the others, with or without pericardial effusion, tamponade, complaints of chest pain and major psychological problems. In asymptomatic patients, the removal of the bullet from the pericardial sac also raises questions ${ }^{[1-3]}$. We report the removal of a floating bullet from the pericardial sac in a 32 year old patient after 3 months of injury.

\section{CASE REPORT}

A 32-year-old laborer was shot by an unknown assailant with a firearm. The weapon had been fired from unknown distance and the bullet entered from the victim's right posterolateral chest wall. According to the patients attendant the patient was escorted to a local hospital where physical examination revealed a puncture wound at the site of entrance of the projectile and there was no exit wound and patient was hemodynamically normal and there was no respiratory distress for this right sided intercoastal tube drainage was done .Patient was then discharged from local hospital on personal request after 7 days of stay during which condition of patient improved. However operation to remove the bullet was not carried out due to refusal of consent for operation by family members. Approximately 2 months and 10 days following his release, he noted shortness of breath and pain in the right side of thorax. He again consulted a local physician and he reffered the patient to the department of cardiothoracic and vascular surgery of S.M.S Medical college and hospital ,Jaipur , Rajasthan, India. Patient was admitted, evaluated and investigated and treatment was started.

$\mathrm{X}$-ray examination revealed a bullet superimposed upon the heart shadow in the area of the left ventricle and no evidence of hemothorax, pneumothorax and pneumoperitoneum was present. X-rays revealed a bullet which freely moved anteriorly, posteriorly, and inferiorly within the pericardium.[Figure 1 - 2] 


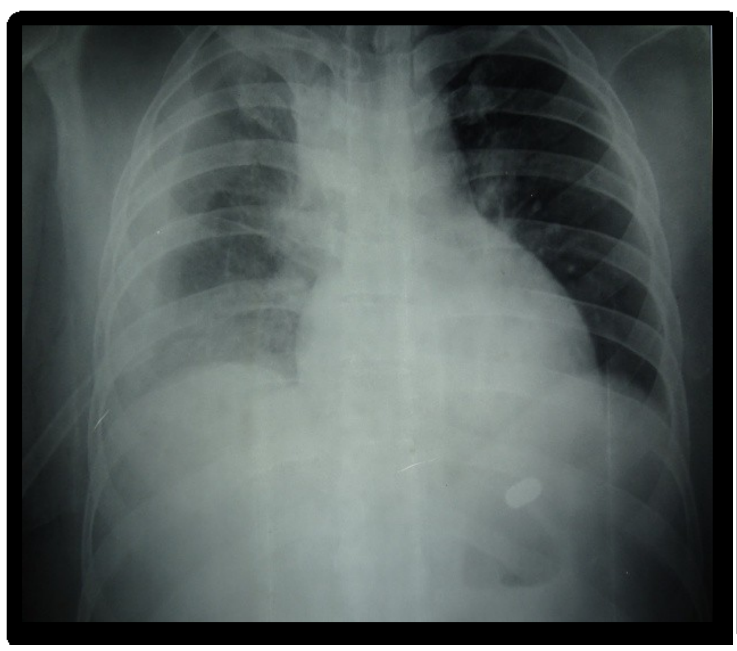

Figure 1

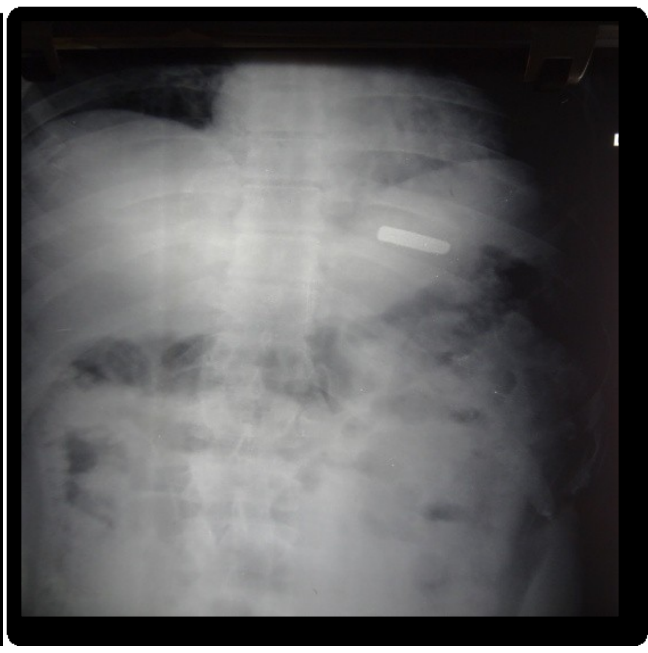

Figure 2

CECT abdomen and chest was done and it gave remarkable finding of large non enhancing hypodense area seen in right lobe of liver measuring approximately $126 \times 113 \times 102 \mathrm{~mm}$ with air loculi and hematoma suggestive of contusion, it was associated with fracture in right posterior inferior ribs with atelectatic changes in right posterior basal segments and ground glass haziness suggestive of lung contusion along with minimal pleural effusion along with circumscribed pericardial effusion of maximum thickness of $16 \mathrm{~mm}$ with metallic foreign body within posterior pericardium measuring approximately $27 \times 11 \mathrm{~mm}$ however no diaphragmatic injury was detected [Fig 3, Fig 4, Fig 5]

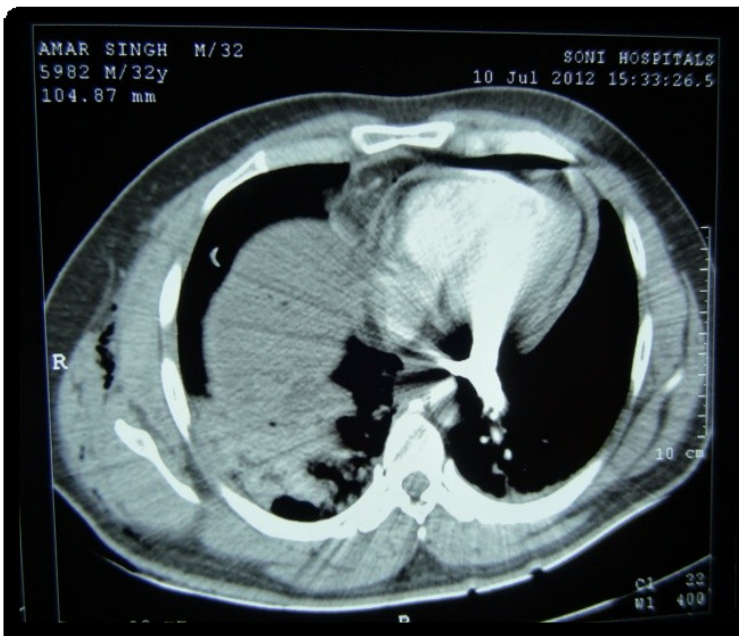

Figure 3

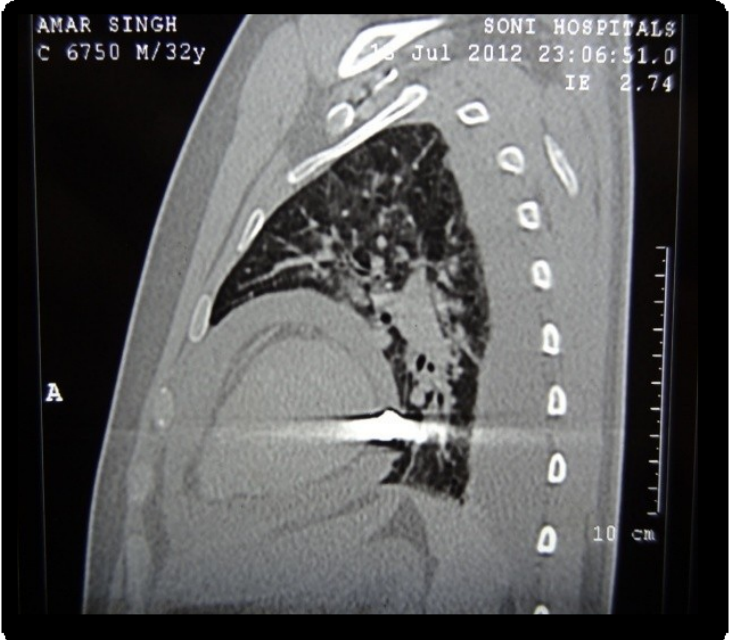

Figure 4

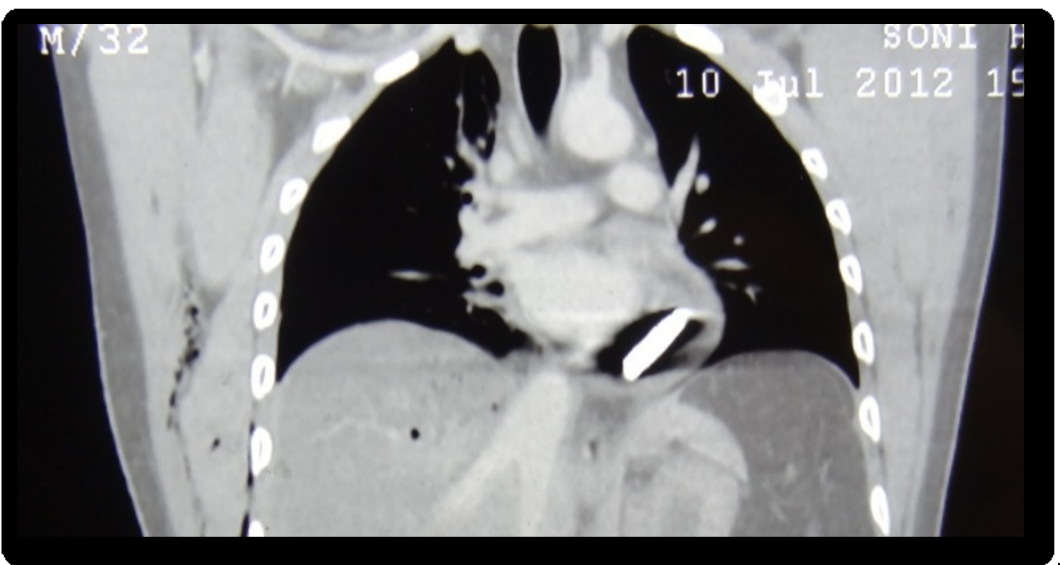

Figure 5 
Patient was planned for elective thoracotomy and removal of bullet .Physical examination revealed an anxious asian male with a pulse of 96 , blood pressure $110 / 68 \mathrm{~mm}$. $\mathrm{Hg}$, a respiratory rate of 28 , and a temperature of $103.2 \mathrm{~F}$. Rales and wheezing were heard at the right base. The remainder of the examination was unremarkable. The following morning the temperature was still $103 \mathrm{~F}$, a soft pericardial friction rub was present, and the hepatojugular reflux test was positive. An electrocardiogram was compatible with acute pericarditis, the neck veins became progressively distended, and the friction rub increased greatly in intensity. A cannula was placed in the right subclavian vein, and the central venous pressure was recorded at $19 \mathrm{~cm}$. of saline. Electrocardiogram was suggestive of sinus tachycardia, rate $110 /$ min, Borderline low voltage of the Q R S complexes in the extremity leads. T h e S-T segment is elevated in leads 2, 3, and A V F and minimally elevated in V4 to V6. Tracing compatible with acute pericarditis.

A decision was made to remove the bullet because of the evidence of cardiac tamponade. The pericardium was explored through a right anteriolateral thoracotomy incision. The pericardium was opaque and inflamed and was $4 \mathrm{~cm}$ thick; $600 \mathrm{ml}$ of serosanguinous fluid was aspirated from the pericardial sac. The undamaged bullet was found in the posterior pericardial sac. A pericardiectomy was carried out. During this procedure the central venous pressure fell from 19 to $10 \mathrm{~cm}$ of saline.

The postoperative course was benign. Culture taken at the time of operation and from the blood on three occasions prior to operation were sterile. The patient continues to do well three months following surgery. [Fig 6 , Fig 7]

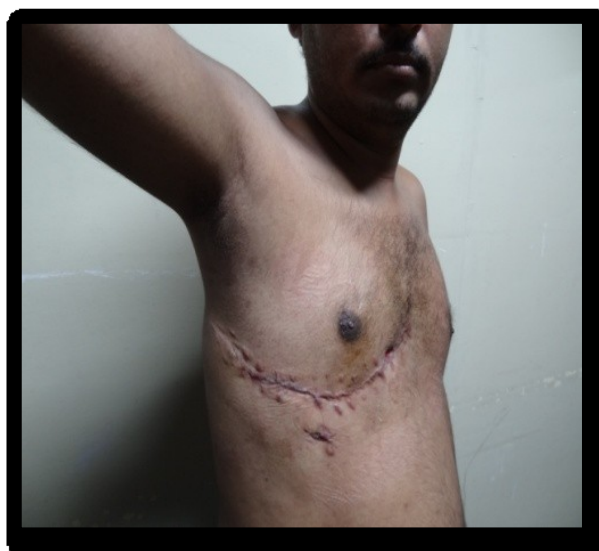

Figure 6

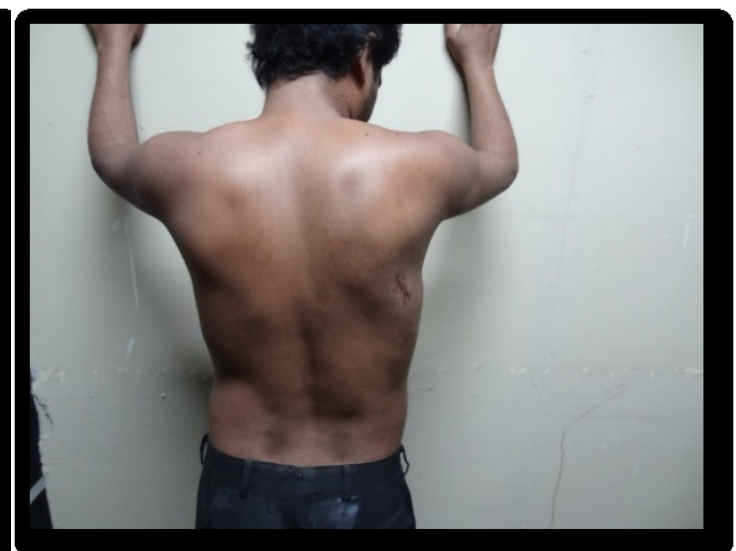

Figure 7

\section{Discussion}

Lower [2] in 1916 described a patient who was shot in the posterior chest with a musket ball. The bullet was seen on $\mathrm{x}$-ray examination to move freely about the pericardial sac with the heartbeat. The patient made an uneventful recovery. Three months later he was readmitted because of shortness of breath and fatigue. ,4 thoracotomy was performed, and the ball was removed. Thereafter the patient was asymptomatic.

Wood [4] in reviewing the British Army Medical Corps experience from World War 11, described a syndrome of acute intermittent pericarditis associated with projectiles in the pericardium. The time of appearance of symptoms varied up to three months following injury. The 5 patients had chest pain, high fever, and signs of acute tamponade. In one instance remission of symptoms occurred in one week. The foreign body was removed in the other 4 patients following which no further episodes of pericarditis occurred.

Valle reported the experience of the Tokyo Army Hospital with pericardial and heart wounds during the Korean War [3]. Of a total 2,811 chest wounds, $4.2 \%$ involved the heart and mediastinum. Forty two patients developed pericardial effusion, usually associated with metallic foreign bodies. These patients experienced precordial pain, fever, tachycardia, and shortness of breath. Pericardial aspiration initially was the treatment employed, and from 300 to $1,800 \mathrm{cc}$ of fluid was aspirated; this material was sterile in $50 \%$ of the cases. Prior to 1953 foreign bodies less than $0.5 \mathrm{~cm}$ in size and not associated with symptoms were not removed. However, 10 patients who were not operated upon and who were returned to duty as cured were readmitted to the hospital because of the recurrence of pericarditis 4 to 26 months later. Only 4 of these patients had positive blood cultures; however, these 4 and 2 others had bacterial growth from the pericardium removed at the time of exploration. On the basis of this experience Valle recommended removal of all foreign bodies in the pericardium, regardless of size. The etiology of recurrent pericarditis is obscure. Certainly in those patients with positive cultures bacterial invasion of the pericardium must be implicated. However, a significant number of such inflammations are bacteriologically negative. The presence of a 
pericardial wound and the foreign body are the common denominators. Foreign bodies enclosed within the myocardium may be associated with recurrent pericarditis.

In Bland and Beebe's survey of 40 patients with myocardial foreign bodies followed for twenty years, 10 developed acute pericarditis which occurred up to four months following injury.

In all cases symptoms disappeared, and no patient developed pericardial calcification or constrictive pericarditis. Bland suggests that this is an example of postpericardiotomy syndrome which he notes to be a poorly understood entity[1]. A similar explanation may be applied to pericarditis in association with pericardial foreign bodies. In these instances, however, the stimulus to pericarditis remains in the form of the projectile. The mechanism involved may be inflammation of the epicardium from the constant motion of the heart against the foreign body and resulting intrapericardial bleeding. Blood in the pericardium is a source of inflammation, and its presence may produce an elevation

\section{Conclusion}

In the case presented in this report, acute pericarditis occurred three months following injury. During a 24-hour period the patient developed the classic signs of pericardial tarnponade, and a pericardiectomy was performed. At the time of the operative procedure the patient was acutely ill with high fever and evidence of cardiac tamponade. It is reasonable to assume that the operative risk of pericardiectomy under these circumstances is much greater than simple removal of a pericardial foreign body. Since the likelihood of recurrent pericarditis is high, all foreign bodies lodged in the pericardium should be removed ${ }^{[7,8,9,10]}$.

Management of such cases generally consists of repairing the injuries occurred during the passage of the bullet. Stress must be placed on the initial resuscitation of the patient and management of critical injuries. Secondly, localization of the bullet should be done. Finally, determination about the removal of bullet should be based on the hazards of its staying in its location versus the hazards of attempt at removal. Because the bullets appear to be out of focus on radiology, one may think they are in the cardiac chambers though the images of bullets retained in the pericardial sac may be blurred due to the spinning effect of the heartbeat on the bullet ${ }^{[5]}$. Both penetrating and blunt cardiac injuries require urgent management. Delayed sequelae and complications have been reported to occur in 4 to $56 \%$ of survivors and frequently required secondary surgical corrections ${ }^{[6]}$ Indeed, the outcome of conservative treatment for intrapericardial foreign bodies is unpredictable, often associated with complications.

\section{REFERENCES}

[1]. Bland, E. F., and Beebe, G. W. Missiles in the heart: A twenty year follow- up of World War I1 cases. N e w Eng. J. M e d. 274:1039, 1966. Friedberg, C. Diseases of the H e a r t (2d ed.). Philadelphia: Saunders, 1956. P. 959.

[2]. Lower, W. E. Removal of bullet from pericardium. Ann. Surg. 63:533, 1916. Nicholson, G. M. Conference of Army Physicians. Central Mediterranean Forces, Rome, 1945

[3]. Valle, A. R. War injuries of heart and mecliastinum. Arch. Surg. 70:398, 1955

[4]. Wood, P. Diseases of the Heart and Circulation (2d ed.). Philadelphia: Lippincott, 1956. P. 927

[5]. Ledgerwood AM. The wandering bullet. Surg Clin North Am1977;57:97-109.

[6]. Szentkereszty Z, Trunqel E, Posan J, Sapy P, Szerafin T, Kiss S. Current issues in the diagnosis and treatment of penetrating chest trauma. Maqy Seb 2007;60:199-204.

[7]. Mattox KL, Limacher MC, Feliciano DV, Colosino L,OMeara ME,Beall AC Jr, DeBakey ME. Cardiac evaluation following heart injury

[8]. Decker HR. Foreign bodies in the heart and pericardium: should they removed? J Thorac Surg. 1939;9(1):62-79

[9]. Burkhart HM, Gomez GA, Jacobson LE, Broadie TA, Tarver RD. Meandering bullet in the pericardial sac: to remove or not remove. Am Surg. 1998;64(4):341-3.

[10]. Watts T, Toone EC. Successful removal of foreign bodies within the pericardium: a report of two cases. Surgery. 1945;17:685-95. 